\title{
Glass valveless micropump using electromagnetic actuation
}

\author{
Christophe Yamahata *, Frédéric Lacharme, Martin A.M. Gijs \\ Institute of Microelectronics and Microsystems, Ecole Polytechnique Fédérale de Lausanne (EPFL), CH-1015 Lausanne, Switzerland
}

\begin{abstract}
We present a valveless micropump in glass, which is magnetically actuated using the sinusoidal current of an external electromagnet. We employ a powder blasting microerosion process for microstructuring the glass substrates and fusion bonding for assembly of the multi-layered microfluidic chip. The reciprocating type micropump contains two nozzle/diffuser elements and a poly(dimethylsiloxane) membrane with embedded permanent magnet. The micropump is self-priming and exhibits a backpressure of $50 \mathrm{mbar}$ and water flow rates up to $1 \mathrm{~mL} / \mathrm{min}$. The flow resonance frequency is in excellent agreement with the model of Olsson et al. [Journal of Micromechanics and Microengineering 9 (1999) 34].
\end{abstract}

(c) 2004 Published by Elsevier B.V.

Keywords: Micropump; Diffuser; Powder blasting; Glass bonding; Electromagnetic actuation

\section{Introduction}

Reciprocating micropumps generally consist of an actuation membrane and two unidirectional valves to convert the periodically applied pressure to a pulsed flow. The two main difficulties in the conception of such micropumps are: (i) the stroke capability of the oscillating diaphragm; (ii) the construction of the valving system. Considering

\footnotetext{
${ }^{*}$ Corresponding author. Tel.: +41 21 6936639; fax: +41 21 6935950.

E-mail address: Christophe.Yamahata@epfl.ch (C. Yamahata).
}

the latter point, Stemme and Stemme [1] developed the concept of valveless reciprocating micropumps, where the valves were replaced by nozzle/ diffuser elements. This solution led to a considerable simplification of the microfabrication process. This work was continued by Olsson [2], who provided a comprehensive study of nozzle/diffuser micropumps and realised different prototypes using both silicon microfabrication technology and thermoplastic replication [3]. We have recently realised an electromagnetically actuated poly(methylmethacrylate) (PMMA) valveless micropump, consisting of two diffuser elements and a poly(dimethylsiloxane) (PDMS) membrane 
with an integrated composite magnet made of $\mathrm{NdFeB}$ magnetic powder [4]. Literature reviews on micropumps discuss the great interest of the nozzle/diffuser valveless solution [5,6]. Most of the developed micropumps - independently of the valving principle used - are silicon-based with piezoelectric actuation. However, the silicon-based micropumps have reduced stroke volume due to the limited deflection capability of the membrane, resulting in a poor tolerance to air bubbles. This brings us to the first mentioned issue which we address with the use of a soft polymeric membrane. The large deflection obtained with electromagnetic actuation results in a high-compression ratio of the chamber and makes our micropump self-priming [7].

Furthermore, chemical inertness and biocompatibility are relevant aspects in the design of micropumps for miniaturized analysis systems. The choice of glass material is particularly attractive for "Lab-on-a-Chip" applications, where miniaturization of laboratory equipment is envisaged. Glass becomes the primary choice when chemical inertness and high-temperature $\left(130^{\circ} \mathrm{C}\right)$ sterilization are required. Therefore, we present a low-cost microfabrication technology for the fabrication of glass microfluidic chips and micropumps, which is based on the micropatterning of glass substrates by powder blasting microerosion. A high-temperature resistant and biocompatible PDMS membrane with embedded permanent magnet is plasma-bonded with the glass structure and gives rise to a large actuation stroke, making the micropump bubble-tolerant and self-priming.

\section{Working principle}

Fig. 1 is a schematic diagram showing the operation principle of our valveless micropump. Electromagnetic actuation is obtained using an external coil, a method previously presented for the PMMA valveless micropump [4]. However, the new designed membrane exhibits a higher stiffness. Pumping pressures obtained here are a factor 4 larger due to the use of an improved actuation coil and a solid magnet rather than a polymer bonded powder magnet. The 4800 turns electromagnet used in the experiments is obtained from Atam Windings s.r.l. (Agrate Brianza, Italy).

In our design of the nozzle/diffuser element (see Fig. 2), the diffuser entrance has rounded corners (curvature radius $r=100 \mu \mathrm{m}$ ) and a width $w_{1}=100 \mu \mathrm{m}$; the outlet has sharp corners and a

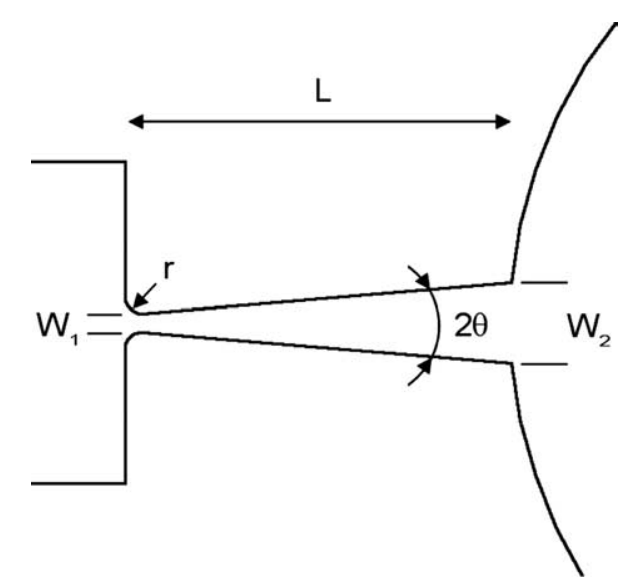

Fig. 2. Definition of the geometrical parameters of the nozzle/ diffuser element.

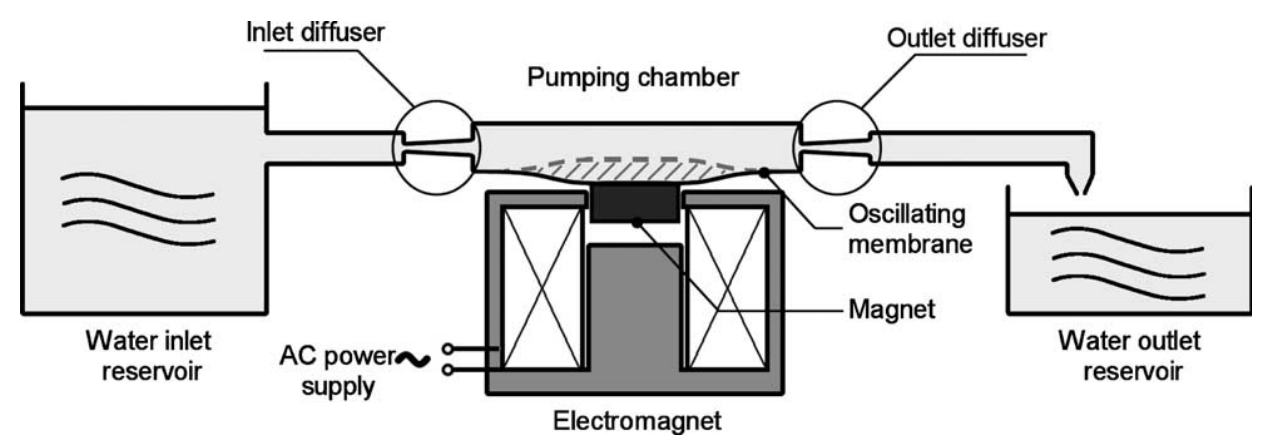

Fig. 1. Schematic diagram of the nozzle/diffuser micropump with external electromagnetic actuation of the magnetic membrane. 


\section{Pre-print version}

width $w_{2}=500 \mu \mathrm{m}$. The diffuser length is $L=2.3 \mathrm{~mm}$, defining the angle $2 \theta=9.5^{\circ}$. The height of the nozzle/diffuser element is $h=300 \mu \mathrm{m}$, as determined by the thickness of the glass plate. The chamber has a diameter of $\varnothing_{\mathrm{m}}=7 \mathrm{~mm}$.

\section{Microfabrication of the micropump}

A schematic burst view of the micropump is shown in Fig. 3. The device consists of three fu-

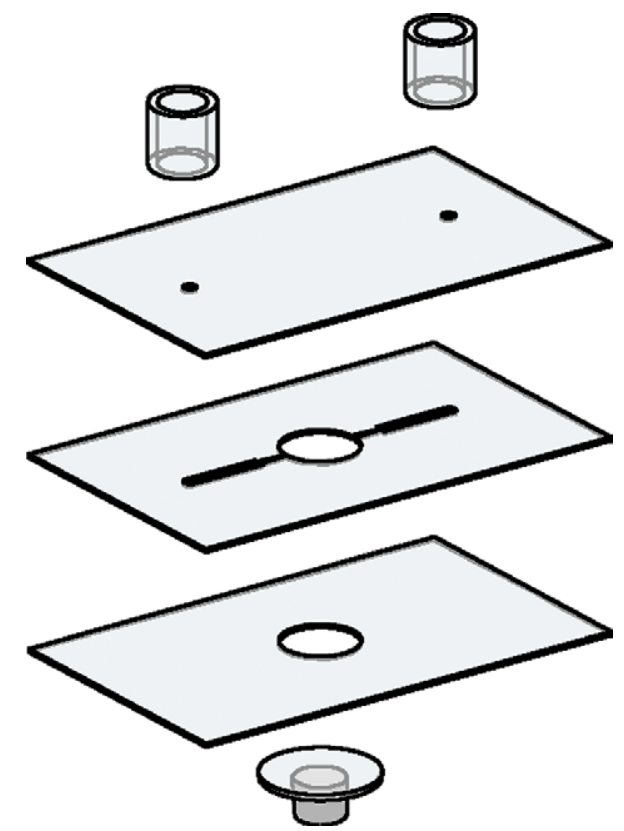

Fig. 3. Burst view of the complete device constituted of three borosilicate glass slides and a flexible PDMS magnetic membrane. sion-bonded glass plates and a PDMS membrane with embedded $\mathrm{NdFeB}$ magnet. Channels and other microfluidic structures of the central layer, as well as the two access holes of the cover glass plate, are fabricated by powder blasting.

\subsection{Silicone magnetic membrane}

Commercially available Sylgard 184 (Dow Corning Corp., Midland, USA) silicone elastomere is used for the fabrication of the membrane. During moulding of the PDMS membrane, we integrate a commercial rare-earth neodymium magnet $(\varnothing 3 \mathrm{~mm} \times 3 \mathrm{~mm} \quad \mathrm{NdFeB} / \mathrm{N} 48$, Maurer Magnetic AG, Grüningen, Switzerland) in the flexible membrane (see Fig. 4).

\subsection{Glass microchannels}

As base material for the realization of the micropumps, we use $300 \mu \mathrm{m}$ thick borosilicate plates (Präzisions Glas \& Optik GmbH, Iserlahn, Germany), protected with metallic masks for microstructuring by powder blasting using small $(30 \mu \mathrm{m})$ alumina particles. After cleaning the glass plates with isopropanol and a piranha solution, the different layers are stacked and aligned together to finally proceed to a high-fusion bonding step of the assembly at $600{ }^{\circ} \mathrm{C}$ [8]. The thermal treatment process is given in Fig. 5 .

Three glass layers are successfully bonded in a single step by this method, a process that can be easily extended to the fabrication of three-dimensional structures with more layers. Fig. 6 shows a diffuser fabricated with this method. The diffuser

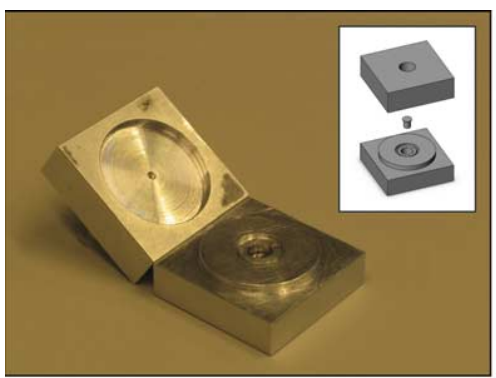

(a)

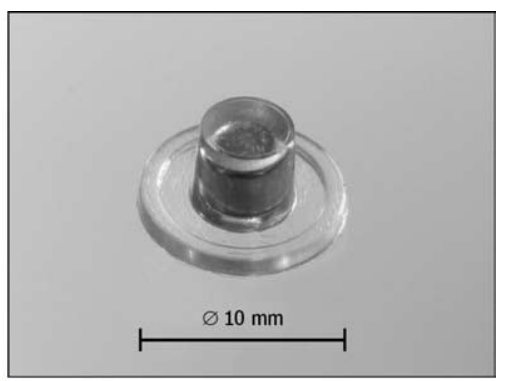

(b)

Fig. 4. (a) Three-part aluminium mould used for the fabrication of the membrane; (b) PDMS membrane with integrated NdFeB magnet (external dimensions: $\varnothing 10 \mathrm{~mm} \times 5 \mathrm{~mm}$ ). 


\section{Pre-print version}

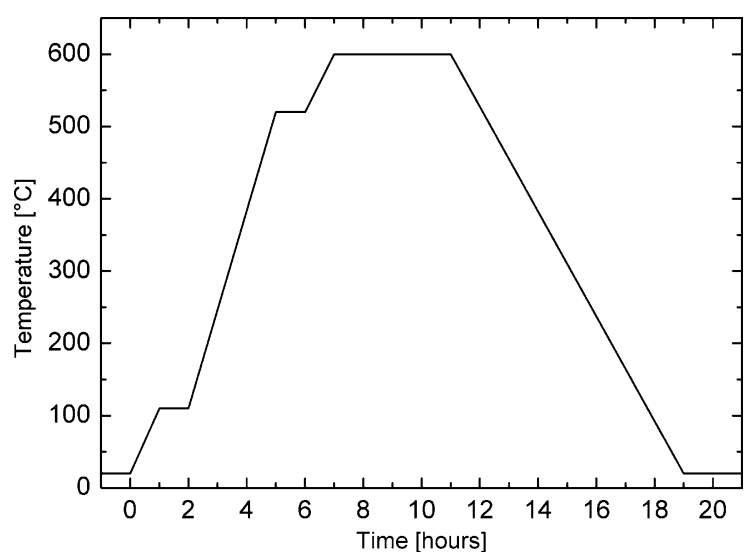

Fig. 5. Thermal treatment for fusion bonding of borosilicate glass substrates.

throat is $w_{1}=100 \mu \mathrm{m}$ wide, which well illustrates the possibilities of the powder blasting microfabrication process.

\subsection{Assembly of the microfluidic chip}

To combine the glass microstructure with the PDMS membrane, the glass surface is treated by an air plasma ('Scancoat Six', BOC Edwards, UK) for $60 \mathrm{~s}$ at $20 \mathrm{~mA}$ and 0.4 mbar. The PDMS membrane is subjected to the same plasma treatment, but for only $10 \mathrm{~s}$. These plasma treatments are sufficient to obtain an irreversible bonding between the glass and the PDMS.

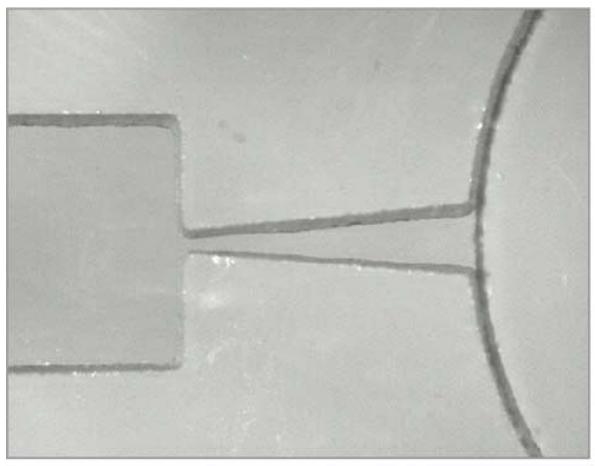

(a)

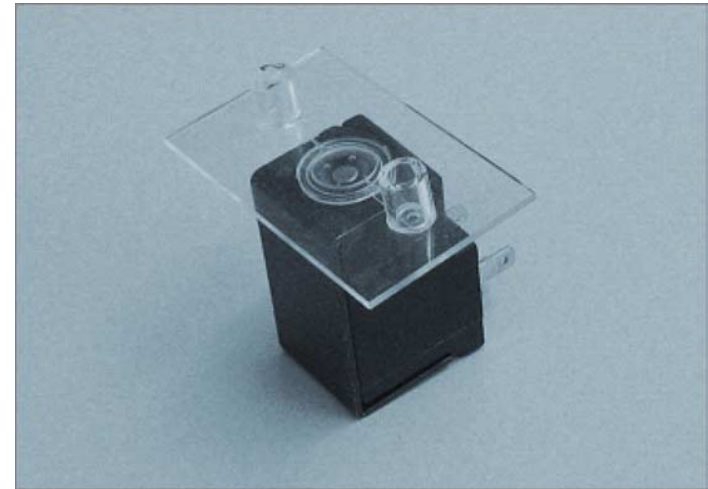

Fig. 7. The glass diffuser micropump with its integrated PDMS magnetic membrane (external dimensions: $36 \mathrm{~mm} \times 22 \mathrm{~mm}$ ). The microchip is placed on top of the electromagnet.

For fluidic connections to the chip surface, cylindrical glass connectors are polished and glued with epoxy solution at $80^{\circ} \mathrm{C}$ for $2 \mathrm{~h}$ (Epo-Tek 3012, Epoxy Technology Inc., MA, USA). A photograph of the finalized micropump together with the actuation electromagnet is shown in Fig. 7.

\section{Characterization of the micropump}

\subsection{Diffuser characteristics}

The fluidic behaviour of the diffuser element was characterized using a separate device. The

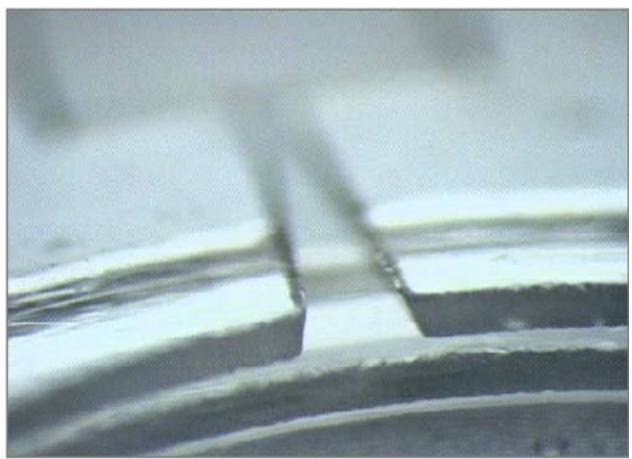

(b)

Fig. 6. (a) Zoom on the inlet diffuser before assembly of the PDMS membrane; (b) side view of the inlet diffuser element after assembly of the glass structure with the PDMS membrane. The glass plates are $300 \mu \mathrm{m}$ thick. 


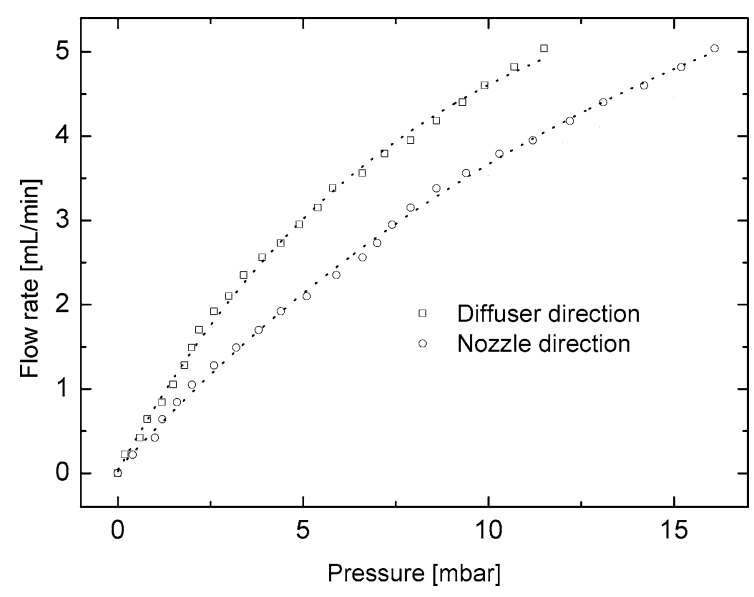

Fig. 8. Water flow rate-pressure characteristics of a discrete nozzle/diffuser element.

dimensions are those defined before (see discussion of Fig. 2), except for the thickness which is $0.5 \mathrm{~mm}$. The efficiency of the nozzle/diffuser element can be derived from Fig. 8 to be $\eta=\left(\phi_{\mathrm{d}} / \phi_{\mathrm{n}}\right)^{2} \approx 2$, with $\phi_{\mathrm{d}}$ and $\phi_{\mathrm{n}}$ the flow rates at a given pressure in the diffuser and in the nozzle direction, respectively.

\subsection{Resonance frequency}

The microfluidic channel depth is $h=300 \mu \mathrm{m}$, the membrane weight is $0.25 \mathrm{~g}$ and its rigidity is measured to be $K=800 \mathrm{~N} / \mathrm{m}$, which corresponds to a diaphragm spring constant $K_{\mathrm{p}}=K / A_{\mathrm{m}}$, with $A_{\mathrm{m}}$ the membrane surface, of $20 \mathrm{MPa} / \mathrm{m}$. Other geometrical dimensions being the same as for the previously developed PMMA valveless micropump, we calculate a theoretical resonance frequency $f_{0} \sim 30-40 \mathrm{~Hz}$ for water using Eq. (1) [9]

$f_{0}=\frac{1}{2 \pi} \sqrt{\frac{K_{\mathrm{p}}(1+\sqrt{\eta})^{2} h\left(w_{2}-w_{1}\right)}{\rho K_{\mathrm{v}}(1+\eta) L \ln \frac{w_{2}}{w_{1}}}}$.

In this equation, $K_{\mathrm{v}}$ is the ratio of the pumping chamber volume variation amplitude to the deflection of the membrane at the center, which we estimated to be $1.96 \times 10^{-5} \mathrm{~m}^{2}<K_{\mathrm{v}}<3.85 \times 10^{-5} \mathrm{~m}^{2}$.

Water flow rate and backpressure characteristics of three nominally identical prototypes are reported in Figs. 9(a) and (b). The experimental results show good reproducibility and the flow resonance frequency is around $50-60 \mathrm{~Hz}$, in good agreement with theory. For a sinusoidal current of $100 \mathrm{~mA}$, we obtain water flow rates of up to 1 $\mathrm{mL} / \mathrm{min}$ and a maximum backpressure of 50 mbar.

\section{Conclusion and outlook}

A valveless glass micropump has been characterized and a low-cost microfabrication process
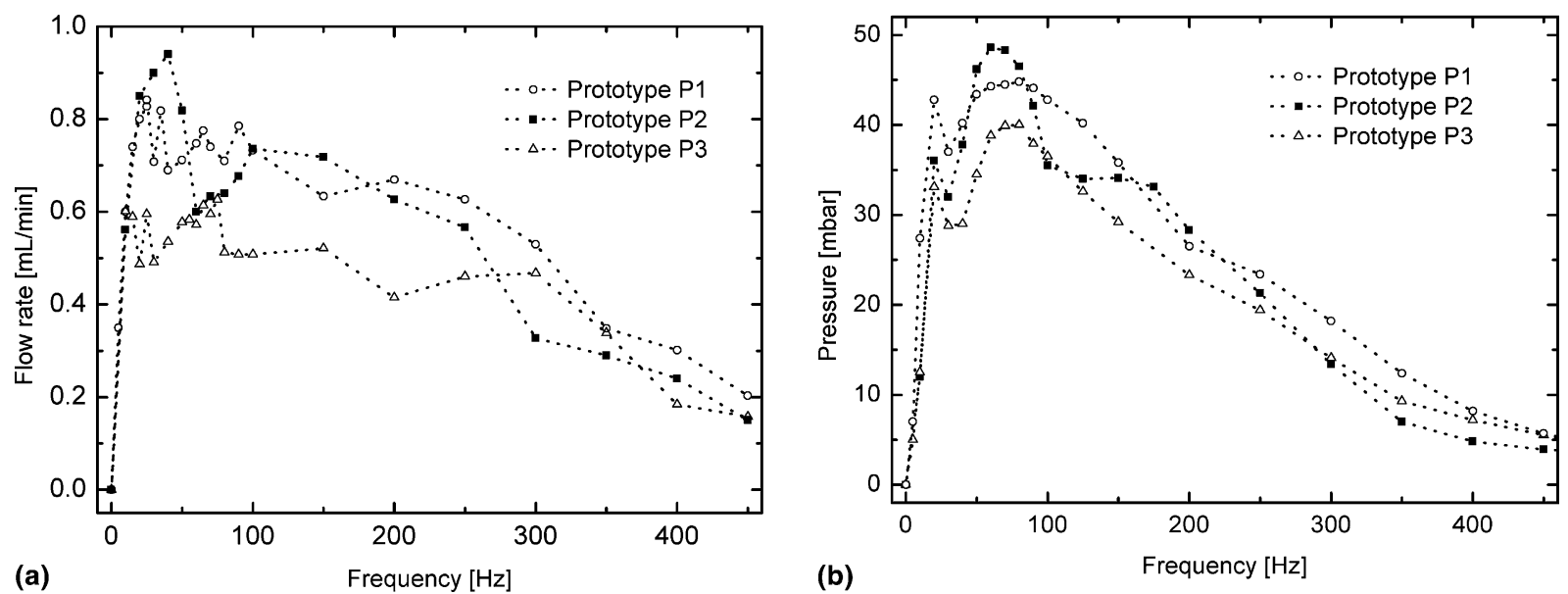

Fig. 9. (a) Water flow rate-frequency and (b) pressure-frequency characteristics of three different prototypes. The experiments are conducted with a $100 \mathrm{~mA}$ sinusoidal current. 


\section{Pre-print version}

has been presented. The results show that glass microstructuring by powder blasting is an interesting method for the fabrication of microfluidic structures. In future, other fluidic elements like ball-valves will be integrated into the micropump design to prevent fluid backflow effects at highbackpressures.

\section{References}

[1] E. Stemme, G. Stemme, Sensors and Actuators A: Physical 39 (2) (1993) 159-167.

[2] A. Olsson, Valve-less diffuser micropumps, Ph.D. Thesis of the Royal Institute of Technology, Stockholm, Sweden, 1998.
[3] A. Olsson, G. Stemme, O. Larsson, J. Holm, L. Lundbladh, O. Ohman, Sensors and Actuators A: Physical 64 (1) (1998) 63-68.

[4] C. Yamahata, C. Lotto, E. Al-Assaf, M.A.M. Gijs, Microfluidics and Nanofluidics. doi:10.1007/s10404-004-0007-6 (in press).

[5] N.T. Nguyen, X.Y. Huang, T.K. Chuan, Journal of Fluids Engineering-Transactions of the ASME 124 (2) (2002) 384 392.

[6] D.J. Laser, J.G. Santiago, Journal of Micromechanics and Microengineering 14 (6) (2004) R35-R64.

[7] M. Richter, R. Linnemann, P. Woias, Sensors and Actuators A: Physical 68 (1-3) (1998) 480-486.

[8] D. Solignac, Glass microchips for bio-chemical analysis: technologies and applications, Ph.D. Thesis of the Swiss Federal Institute of Technology, Lausanne, Switzerland, 2003.

[9] A. Olsson, G. Stemme, E. Stemme, Journal of Micromechanics and Microengineering 9 (1) (1999) 34-44.

\section{This article is available online at the following address:}

doi: 10.1016/j.mee.2004.12.018 